Cahiers $d u$ MONDE RUSSE

\section{Cahiers du monde russe}

Russie - Empire russe - Union soviétique et États indépendants

$62 / 4 \mid 2021$

Varia

\title{
Mie NAKACHI, Replace the Dead. The Politics of Reproduction in the Postwar Soviet Union
}

\section{Alain Blum}

\section{(2penEdition}

\section{Journals}

Édition électronique

URL : https://journals.openedition.org/monderusse/12832

DOI : $10.4000 /$ monderusse. 12832

ISSN : $1777-5388$

\section{Éditeur}

Éditions de l'EHESS

\section{Édition imprimée}

Date de publication : 1 décembre 2021

Pagination : 717-720

ISBN : 978-2-7132-2895-7

ISSN : $1252-6576$

Référence électronique

Alain Blum, "Mie NAKACHI, Replace the Dead. The Politics of Reproduction in the Postwar Soviet Union », Cahiers du monde russe [En ligne], 62/4 | 2021, mis en ligne le 01 décembre 2021, consulté le 16 septembre 2022. URL : http://journals.openedition.org/monderusse/12832 ; DOI : https://doi.org/ 10.4000/monderusse.12832

Ce document a été généré automatiquement le 16 septembre 2022.

Tous droits réservés 


\title{
Mie NAKACHI, Replace the Dead. The Politics of Reproduction in the Postwar Soviet Union
}

\author{
Alain Blum
}

\section{RÉFÉRENCE}

Mie NAKACHI, Replace the Dead. The Politics of Reproduction in the Postwar Soviet Union, New York : Oxford University Press, 2021, p. 328

Il peut paraître étrange de choisir une loi pour « héroïne » d'un ouvrage qui contribue à l'histoire soviétique de l'après seconde guerre mondiale. D'autant plus étrange que cette loi, publiée au milieu de l'année 1944, porte sur la famille, alors que l'URSS fait face à bien d'autres enjeux et obstacles et à une reconquête suivie d'une sortie de guerre particulièrement douloureuse. Or, dans son passionnant ouvrage, Mie Nakachi fonde une grande partie de sa recherche sur la loi « Sur l'augmentation du soutien aux femmes enceintes, aux mères de famille nombreuse, aux mères isolées, et sur le renforcement de la protection de la maternité et de l'enfance ; sur l'établissement du titre honorifique de "Mère Héroïne", la création de l'ordre de la "Gloire à la maternité", et la " Médaille à la maternité" ", promulguée le 8 juillet 1944. Le choix de l'auteure de partir de ce texte, ou plus précisément de son élaboration, est très judicieux, car cela lui permet d'écrire bien plus qu'une histoire de la politique démographique en URSS. Elle traite tant des processus de décision dans l'URSS stalinienne, des conséquences sociales d'une simple loi, que des conséquences démographiques et sociales de la seconde guerre mondiale et de l'immensité du déséquilibre entre hommes et femmes qui en résulta; enfin elle s'intéresse aux transformations qui traversèrent l'Union soviétique de l'après-guerre à aujourd'hui, sans faire de la mort de Stalin une rupture radicale. 
2 L'ouvrage débute par l'étude de l'élaboration de cette loi aux dimensions natalistes prédominantes, même si, selon les destinataires, la propagande veut en faire une loi sociale, protégeant mères et enfants. Hruščev qui prend l'initiative de ce texte, propose dès le début deux versions justifiant le projet de loi : l'une met en avant le souci de protéger mères et enfants à la sortie de la guerre, l'autre révèle au contraire la dimension presque exclusivement nataliste du projet, en réponse au drame humain que l'URSS a subi. Mie Nakachi étudie alors le processus décisionnel auquel ont participé de très nombreux acteurs (ministères en particulier) entre la première proposition et l'établissement $\mathrm{du}$ texte définitif. Elle nous propose une étude novatrice des négociations et interventions dans un tel processus, en pleine sortie de guerre. Elle examine les conséquences de ces logiques natalistes qui mettent en avant l'imposition des personnes sans enfants, renforcent et modifient la nature d'une mesure décrétée dès 1941 et déjà à l'initiative de Hruščev : "Le célibat et la famille de taille réduite furent, pour la première fois, considérés comme une offense portée à l'État, à laquelle il fallait répondre avec sévérité » (p. 34). Outre la lutte renforcée contre l'avortement, le projet de loi proposé par Hruščev encourageait de façon très particulière les naissances hors mariage, reconnaissant de façon non équivoque les conséquences de l'immense déficit de jeunes hommes. Le projet initial affirme que l'État assurera le soutien des enfants ainsi nés et, par exemple, donnera le «droit » d'abandonner l'enfant dans un orphelinat, l'État se substituant à la mère et au père. La loi finalement adoptée distingue clairement les naissances hors mariage des naissances issues du mariage, alors que cette distinction avait été abolie après la révolution de 1917. Afin de dégager le père de toute obligation de soin et de reconnaissance paternelle et empêcher une mère de se pourvoir en justice à ce propos, la loi n'autorise à donner le patronyme et le nom de famille du père à l'enfant que dans le cas d'un couple marié. En revanche, sur la pression du ministère de la Santé et dans un contexte où l'avortement était devenu courant durant la guerre, le renforcement des mesures répressives contre l'avortement ne sont pas retenues.

3 L'ouvrage de Mie Nakachi explore ensuite les transformations des pratiques et des situations sociales qui, bien que de façon de plus en plus atténuée, font encore écho aux conséquences de la guerre et suivent ce nouveau cadre législatif. L'auteure illustre la tension entre les différents acteurs intervenant dans les débats, que ce soient les acteurs institutionnels - ministère de la Santé, direction de la statistique, ministère de la Justice -, ou les acteurs individuels - médecins, journalistes ainsi que les femmes qui, par leurs lettres, réclament des changements. Les nombreux débats et discussions au sommet, mais aussi les interventions publiques autour de la question de l'avortement, qu'analyse Mie Nakachi, sont extrêmement éclairants sur les mécanismes de pouvoir, sur l'action de différents milieux, mais aussi sur l'agentivité des femmes, directement concernées par ces questions. L'auteure montre comment les positions officielles et certains acquis peuvent être brutalement annihilés par les combats politiques menés par Stalin, sans rapport direct avec la question, mais qui n'en n'ont pas moins de lourdes conséquences. Ainsi, malgré les dispositions législatives, la position relativement ouverte vis-à-vis de l'avortement est brutalement remise en question par la purge du milieu médical qui débute en 1946 et frappe en particulier l'Institut d'obstétrique de Moscou. Le suivi de ces politiques avant et après la mort de Stalin, les positions de la ministre de la Santé, Kovrigina, qui, déjà en place après la guerre, le demeure après la mort de Stalin, celles de Hruščev qui continue à défendre une politique nataliste appuyée, mais est contraint de renoncer à la criminalisation de 
l'avortement alors qu'il en avait demandé en 1944 le renforcement, révèlent la complexité des positions et des débats qui font suite à 1953.

4 Enfin, Mie Nakachi s'intéresse aux transformations plus tardives, alors que les stigmates de la seconde guerre mondiale se sont estompés, mais que l'enjeu nataliste reste bien présent. Elle montre qu'en dépit de ce qu'aurait pu laisser espérer l'abolition de la criminalisation de l'avortement, de nombreuses années seront nécessaires avant que les diverses mesures de la loi de 1944 ne soient définitivement mises de côté. L'hostilité de Hruščev à toute modification, bien que contraint, malgré lui, à décriminaliser l'avortement, l'explique partiellement. Il faut attendre 1968, soit longtemps après son éviction, pour qu'une nouvelle loi soit promulguée, alors que des interventions publiques avaient réclamé, dès après la mort de Stalin, une législation protégeant mieux les femmes, interventions largement publiées dans des revues telle la Literaturnaja Gazeta.

5 Cet ouvrage n'est pas simplement celui de l'histoire de prises de décisions politiques et des débats et autres polémiques qui entourent ces questions. Les conséquences sociales des diverses décisions prises, mais aussi l'impact de la société sur ces décisions, sont bien présents. Cette histoire sociale met au premier plan les relations genrées, les conséquences sociales de la loi de 1944, puis de son abandon partiel en 1955. Elle montre comment le très fort appui à la mise au monde d'enfants hors mariage, dont le père n'est pas reconnu comme tel, a de profondes répercussions sur la situation des femmes. "La politique familiale, du point de vue de l'État soviétique, ayant créé des millions d'enfants sans pères, pouvait être considérée comme un succès. Cependant, cette "réussite" de l'État avait provoqué un important mécontentement, notamment parmi les femmes. » (p. 115) «Le gouvernement soviétique avait abaissé le statut de la femme après la guerre, identifiant la reproduction féminine à un outil destiné à rétablir l'économie et la société soviétiques, manipulant les lois sur le mariage et le divorce pour rendre plus facile aux hommes d'envisager la reproduction, créant ainsi un groupe stigmatisé d'enfants nés hors mariage et de mères célibataires, sans offrir un soutien suffisant aux mères ayant mis au monde seule un enfant » (p.122).

6 Mie Nakachi décrit aussi les immenses conséquences, directes, de la guerre sur les relations de genre, que le législatif a amplifiées. Les couples éphémères formés sur le front, les veuves de guerre extrêmement nombreuses, rendent encore plus difficile la condition des femmes, difficultés renforcées par la loi qui, outre cet "abandon » des femmes avec enfants a engendré de nombreux conflits juridiques pour les couples non mariés avant 1944 et posé bien d'autres questions. «Finalement, l'adoption de la loi a provoqué de forts conflits d'intérêt genrés, les mères se précipitant pour enregistrer leur mariage, et les pères, convaincus que le soutien apporté aux enfants était désormais à charge de l'État, refusant cette responsabilité. La présence d'une autre famille, la désaffection vis-à-vis de l'épouse après la longue absence pendant la guerre, le pur égoïsme, et diverses combinaisons de ces facteurs firent obstacle à la résolution du profond clivage, provoqué par la loi de 1944 sur la famille» (p. 101).

7 Enfin, Mie Nakachi montre à quel point la loi de 1968 sur la famille persistait dans une vision très genrée, «les hommes conservant la possibilité de ne pas reconnaître leur enfant né hors mariage, la situation des femmes changeant pour le pire ».

8 L'auteure parcourt ensuite très rapidement les années qui suivent. L'épilogue souligne la résurgence d'une politique nataliste sous la présidence Putin, sans pour autant 
approfondir les relations qui pourraient être établies entre l'histoire que Mie Nakachi analyse en profondeur et les dynamiques contemporaines.

Les sources consultées par l'auteure sont essentiellement conservées au GARF et au RGASPI, mais soulignons aussi la richesse du fonds de la ministre de la Santé, Kovrigina, conservées au sein du musée des collections privées - Archives centrales de Moscou (CMA-MLS). De très nombreuses lettres de femmes présentes dans ce fonds, contribution essentielle à une histoire des relations de genre, ont permis à l'auteure de toucher au plus près une histoire des femmes et de leurs interventions et influences sur les transformations des politiques démographiques et sociales.

Mie Nakachi nous offre là un très bel ouvrage, dont l'intérêt dépasse très largement l'histoire de la famille ou l'histoire de la population. En retraçant une histoire qui ne fait pas de la mort de Stalin une rupture radicale, mais qui contribue à rappeler que de nombreux débats étaient déjà présents avant 1953 et ont été concrétisés par des décisions au-delà de cette date, elle éclaire d'un jour nouveau les processus de décision dans l'URSS stalinienne d'après-guerre. Elle propose une histoire sociale, une contribution à une histoire des femmes de l'après-guerre à aujourd'hui, tout en ouvrant de nombreuses pistes de recherche.

\section{AUTEURS}

\section{ALAIN BLUM}

CERCEC, EHESS

INED 